\title{
An irew1 mutant intensifies lateral root branching for adaption upon water stress
}

\section{Suchang Huang' \& Rui Miao*,1}

${ }^{1}$ College of Life Sciences, Fujian Agriculture \& Forestry University, Jinshan Fuzhou 350002, China; *Author for correspondence: miaorui2011@126.com

BioTechniques 69: 243-244 (October 2020) 10.2144/btn-2020-0053

First draft submitted: 24 April 2020; Accepted for publication: 28 May 2020; Published online: 26 June 2020

\section{KEYWORDS}

irew1 • lateral root $\bullet$ lateral root primordia $\bullet$ root system architecture $\bullet$ water stress

Drought is an environmental threat for agriculture [1]. As climate change continues to heat and dry the world, we are forced to breed more water-saving and high-yielding crops [2]. Plants have developed sophisticated mechanisms to use water efficiently. The plant root is the most important organ to absorb water in soil and root system architecture (RSA) and tropisms are critical strategies to improve plant drought tolerance. RSA consists of the primary root (PR) and lateral root (LR); LR formation plays a central role in this adaptive system [3]. The molecular and cellular mechanism of LR development has been described in most detail in Arabidopsis [4]. LR development can be divided into two phases - LR initiation and LR emergence - and demonstrates eight stages of development of primordia [5]. Environmental inputs affect LR branching and plants produce various RSAs in response to environmental stimuli [6,7]. For example, in order to use water efficiently, plants alter RSA to grow shallowly or deeply upon environmental stimuli. Shallow RSAs with increased LR branching are good for capturing water from moist soil at the surface, whereas RSAs with increased PR length and steepness, accompanied by reduced LR numbers and branching angles, can be beneficial for the capture of water from soil in the deep moisture layers when the soil surface is dry [8]. Another useful strategy for water efficiency is root hydrotropism, in which roots chase water toward soil moisture in any direction [8-10]. Plants should highly integrate RSA and hydrotropism to survive under water-deprived conditions.

We previously isolated an Arabidopsis 'improved root elongation under water stress 1' (irew1) mutant that noticeably promotes PR elongation, but not root hydrotropism, under water stress medium (WSM) [11]. Interestingly, irew1 might stimulate the stress hormone abscisic acid ( $A B A$ ) response; extra application of $\mathrm{Ca}^{2+}$-enhanced PR elongation in irew1 mutant and $\mathrm{ABA}$ biosynthesis-deficient mutants ABA deficient 1 (aba1-1) and ABA deficient 2 (aba2-1), but not in an ABA receptor sextuple mutant pyr1pyl1pyl2pyl4pyl5pyl8 (112458) under WSM, indicating that $\mathrm{Ca}^{2+}$ signaling intersects with the ABA signal transduction pathway for root adaption upon water stress [11]. In this study, we analyzed the characteristics of RSA of an irew1 mutant that displays enhanced tolerance to water stress.

To examine the characteristics of the irew1 mutant's RSA, we assessed the formation and development of lateral root primordia (LRP) and calculated the LR emergence rates in irew1 mutant seedlings at 7 days after germination. Seeds of Arabidopsis thaliana ecotype Columbia-0 (Col-0) wild-type and irew1 mutant were first surface sterilized with 100\% bleach for 3 min, washed five-times with sterile water and then sown on plates containing half-strength Murashige and Skoog medium supplemented with 1\% (w/v) sucrose and $0.8 \%(\mathrm{w} / \mathrm{v})$ agarose. irew 1 is in Col-0 background. Plates were kept at $4{ }^{\circ} \mathrm{C}$ for 2 days for vernalization, then the materials were grown vertically for 5 days and transferred to half-strength Hoagland's medium (normal medium, NM) and half-strength Hoagland's medium containing $0.3 \%$ glycerol in a water potential gradient assay for 2 days as described by Miao et al. [9]. The plants were placed in growth chambers under dark-light cycles $\left(8 \mathrm{~h}\right.$ dark $\left[21^{\circ} \mathrm{C}\right]$ and $16 \mathrm{~h}$ light $\left.\left[23^{\circ} \mathrm{C}\right]\right)$. LR numbers were calculated on digital images of the plastic square plates by ImageJ 1.40 software [12]. The number of LRP was counted using a Nikon Eclipse E100 microscope. The clearing of Arabidopsis roots for LRP calculation was performed according to the protocol described by Malamy and Benfey [13].

Interestingly, irew1 mutants displayed increased LR emergence rate compared with the wild-type Col-0 in the normal growth condition (NM), although the total number of LRP in the irew1 mutant was similar to that in Col-0 in NM (Figure 1A-C), indicating that the irew1 mutant produced more LR than the wild-type using the same amount of LRP. On the other hand, the wild-type Col-0 displayed fewer LR and LRP under WSM and the LR emergence rate under WSM was similar to that of Col-0 in NM. However, the irew1 mutant exhibited similar numbers of LRP in NM and WSM but produced more LR upon WSM than in NM; thus the irew1 mutant could access more water and demonstrated a better adaption capability in response to water-deficient conditions (Figure 1C \& D).

To further investigate the mechanism of the irew1 mutant, we extracted total RNA from the roots of irew1 mutant and wild-type Col0 seedlings at 7 days after germination in NM and WSM, using the TRIzol reagent (Beijing TransGen Biotech Co., Ltd, Beijing China) in accordance with the manufacturer's protocol according to Yuan et al. [11]. The RNA-Seq data used in the study was deposited in the Gene Expression Omnibus [14] under code PRJNA605515. The number of downregulated root development-related differentially expressed genes for Col-0-NM versus Col-0-WSM was more than that of irew1-NM versus irew1-WSM, suggesting that irew1 preserved root 
(A)

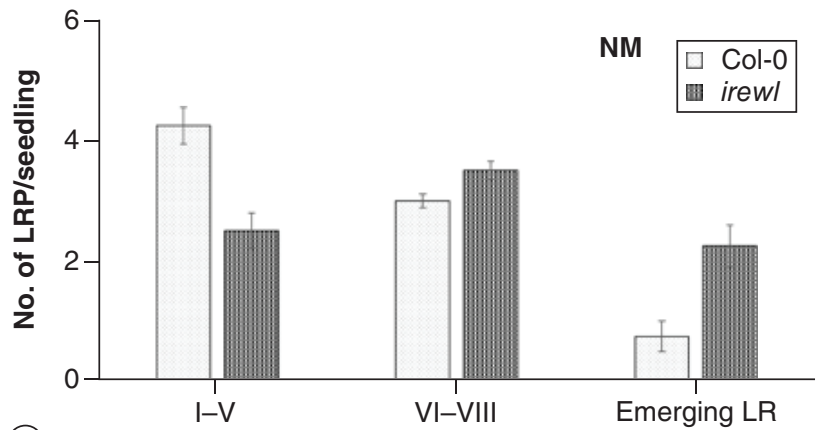

(B)

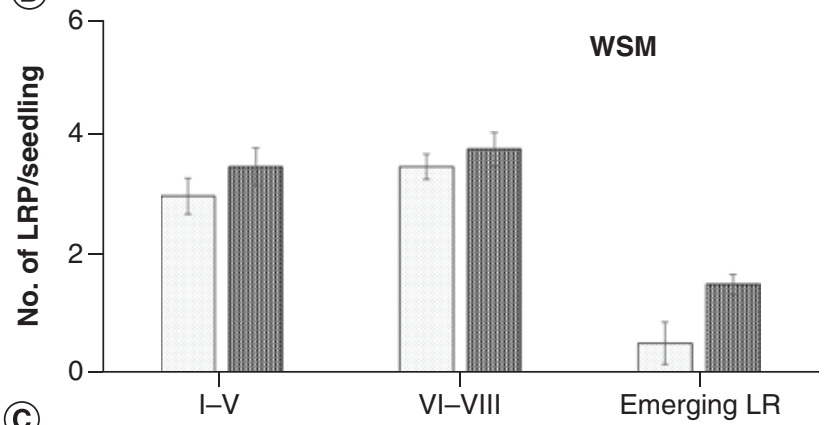

(C)

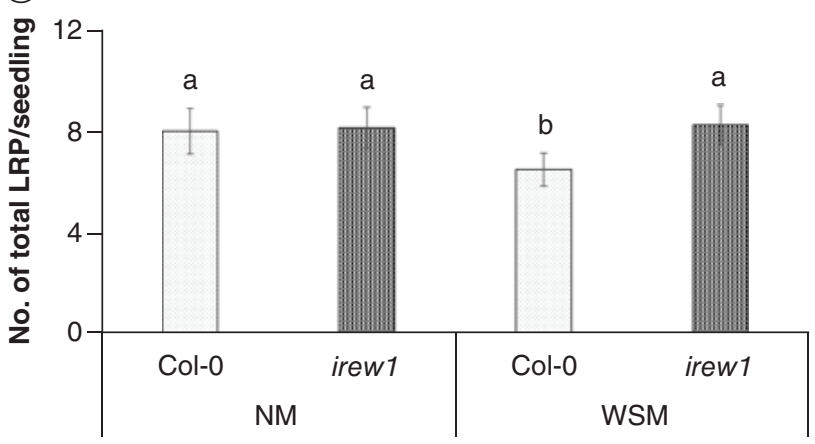

(D)

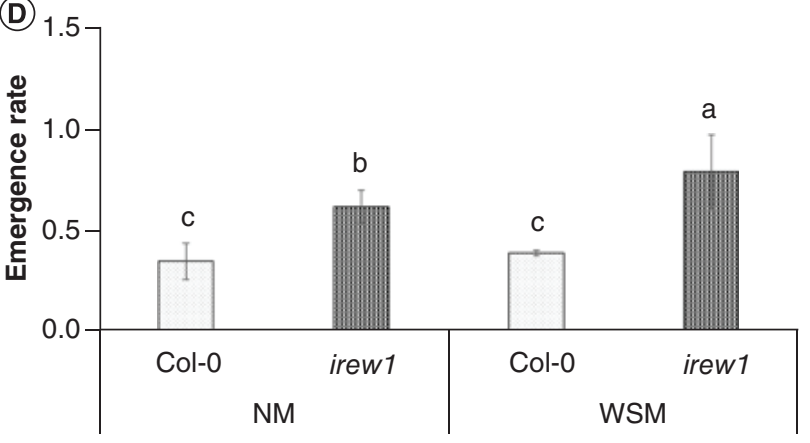

(E)

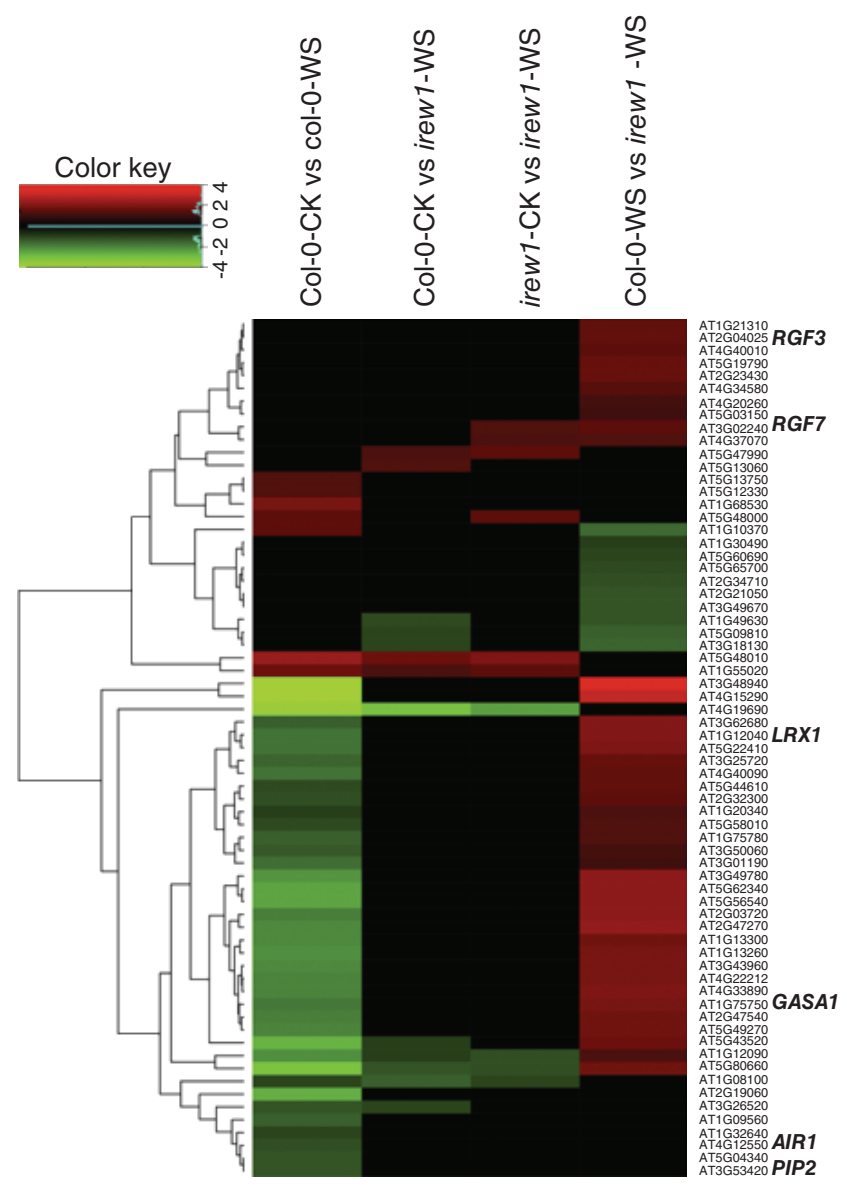

(F)

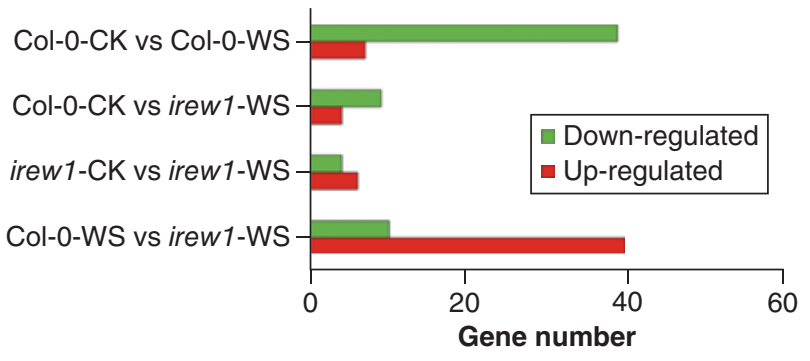

Figure 1. irew1 mutant sustains the expression of root development-related genes and triggers lateral root branching under water stress. (A \& B) The LRP numbers of the wild-type Col-0 and irew1 mutant grown in NM (A) and WSM (B). (C) The total LRP of the wild-type Col-0 and irew1 mutant in NM and WSM. (D) The LR emergence rate of the wild-type Col-0 and irew1 mutant in NM and WSM. The values are means and error bars show \pm SD of 12 seedlings from three independent experiments $(n=36)$. (E) Heat map chart derived from hierarchical cluster analysis of root development-related genes that emerged as altered in RNA-Seq results. Each row represents a gene; columns indicate Col-0-WSM versus Col-0-NM, Col-0-NM versus irew1-WSM, irew1-NM versus irew1-WSM and Col-0-WSM versus irew1-WSM, respectively. Red and green indicate increase and decrease, respectively, of the levels of gene expression compared with the unstimulated control, while black indicates no change. (F) Number of root development-related genes in Col-0-WSM versus Col-0-NM, Col-0-NM versus irew1-WSM, irew1-NM versus irew1-WSM and Col-0-WSM versus irew1-WSM. Downregulated and upregulated genes are shown in green and red, respectively.

Col-0: Columbia-0; LRP: Lateral root primordia; NM: Normal medium; SD: Standard deviation; WSM: Water stress medium. 
development-related gene expression under WSM (Figure 1F). In the transcriptome analysis, interestingly, we found that the expression level of rgf7 (AT3G02240), encoding a root meristem growth factor, was upregulated in irew1, but not in Col-0, in response to water stress (Figure 1E). rgf7 is a member of the secreted peptides family and is expressed mainly in the innermost layer of central columella cells, which are required for conservation of the root stem cell niche and the proliferation of transit-amplifying cells [15,16]. As for rgf7, the expression level of rgf3 (AT2G04025) in irew1 was also found to be higher than that of Col-0. Conversely, the expression of multiple genes related to LR growth and development was downregulated in Col-0 and unchanged in irew1 (Figure 1E); such genes included air1 (AT4G12550), Irx1 (AT1G12040), gasa1 (AT1G75750) and pip2 (AT3G53420). Taken together, RNA-Seq data revealed that the irew1 mutant optimized the expression profiles of root-related genes and sustained root growth under water stress.

\section{Conclusion}

The irew1 mutant might intensify LR formation to expand the effective water absorption area for root adaption in water-deficient conditions.

\section{Author contributions}

S Huang and R Miao carried out all the experiments and the majority of the data analysis. R Miao completed the data analysis, performed statistical analysis and wrote the manuscript.

\section{Financial \& competing interests disclosure}

The authors are grateful for grant support from the National Natural Science Foundation of China (no. 31600209). The authors have no other relevant affiliations or financial involvement with any organization or entity with a financial interest in or financial conflict with the subject matter or materials discussed in the manuscript apart from those disclosed.

No writing assistance was utilized in the production of this manuscript.

\section{Open access}

This work is licensed under the Attribution-NonCommercial-NoDerivatives 4.0 Unported License. To view a copy of this license, visit http://creativecommons.org/licenses/by-nc-nd/4.0/

\section{References}

Papers of special note have been highlighted as: • of interest; $\bullet \bullet$ of considerable interest

1. Food and Agriculture Organization of the United Nations. The impact of disasters and crises on agriculture and food security. http://www.fao.org/family-farming/detail/en/c/1110639/

2. Food and Agriculture Organization of the United Nations. Proactive approaches to drought preparedness - where are we now and where do we go from here? http://www.fao.org/in-actio $\mathrm{n} /$ kore/publications/publications-details/fr/c/1258929/

3. Zhao Y, Xing L, Wang X et al. The ABA receptor PYL8 promotes lateral root growth by enhancing MYB77-dependent transcription of auxin-responsive genes. Sci. Signal. 7(328), ra53 (2014).

4. Ditengou FA, Teale WD, Kochersperger P et al. Mechanical induction of lateral root initiation in Arabidopsis thaliana. Proc. Natl Acad. Sci. USA 105(48), 18818-18823 (2008).

5. Laskowski M, Williams M, Nusbaum H et al. Formation of lateral root meristems is a two-stage process. Development 121(10), 3303-3310 (1995).

6. Malamy JE, Ryan K. Environmental regulation of lateral root initiation in Arabidopsis. Plant Physiol. 127, 899-909 (2001).

7. Malamy JE. Intrinsic and environmental response pathways that regulate root system architecture. Plant Cell Environ. 28(1), 67-77 (2005).

8. Gupta A, Rico-Medina A, Caño-Delgado Al. The physiology of plant responses to drought. Science 368(6488), 266-269 (2020).

9. Miao R, Wang M, Yuan W et al. Comparative analysis of Arabidopsis ecotypes reveals a role for brassinosteroids in root hydrotropism. Plant Physiol. 176(4), 2720-2736 (2018).

- Describes the main method used in this study.

10. Yuan W, Li Y, Li L et al. BR-INSENSITIVE1 regulates hydrotropic response by interacting with plasma membrane $\mathrm{H}^{+}$-ATPases in Arabidopsis. Plant Signal. Behav. 13(8), e1486147 (2018).

11. Yuan W, Zhang Q, Li Y et al. Abscisic acid is required for root elongation associated with Ca2+ influx in response to water stress. Front. Plant Sci. 11, 332 (2020).

-. Characterizes the irew1 mutant.

12. ImageJ 1.40 software. http://rsb.info.nih.gov/ij/

13. Malamy JE, Benfey PN. Organization and cell differentiation in lateral roots of Arabidopsis thaliana. Development 124, 33-44 (1997).

14. Gene Expression Omnibus. http://www.ncbi.nim.nih.gov/geo/

15. Matsuzaki Y, Ogawa-Ohnishi M, Mori A et al. Secreted peptide signals required for maintenance of root stem cell niche in Arabidopsis. Science 329(5995), 1065-1067 (2010).

16. Meng L, Buchanan BB, Feldman LJ et al. CLE-like (CLEL) peptides control the pattern of root growth and lateral root development in Arabidopsis. Proc. Natl Acad. Sci. USA 109(5), 1760-1765 (2012). 
